\title{
Telepathology in the Philippines: A Review and Future Prospects
}

\author{
Elizabeth Arcellana-Nuqui, ${ }^{1}$ Paul A. Fontelo ${ }^{2}$ and Alvin B. Marcelo ${ }^{3,4}$ \\ ${ }^{1}$ The Medical City, Ortigas Avenue, Pasig City \\ ${ }^{2}$ Lister Hill National Center for Biomedical Communications, National Library of Medicine, National Institutes of Health in Bethesda, Maryland, USA \\ ${ }^{3}$ Medical Informatics Unit, University of the Philippines Manila \\ ${ }^{4}$ Department of Surgery, College of Medicine and Philippine General Hospital, University of the Philippines Manila
}

\begin{abstract}
Background. Challenged with insufficient number and maldistribution of pathologists, the Philippines seemed poised to benefit from telemedicine.
\end{abstract}

Methods. The first modern Internet-based telepathology consultation was conducted between the University of the Philippines Manila and the Armed Forces Institute of Pathology in 1997.

Results. While the first remote telepathology consult was a success, more than a decade after this historic referral, telepathology remains in the fringes of mainstream pathology practice despite its huge potential to benefit the underserved population. Challenges with human, organizational, and technology factors hinder its progress.

Conclusions. While the original consultation faced difficulties in human capacity, connectivity and infrastructure, rapid developments in governance and technology have the potential to eliminate these problems. Substantial improvements in recent years now provide a more conducive environment to deliver telepathology services to remote areas. This paper proposes a framework for the establishment of mature telepathology services to enable its use in areas of greatest need in the country.

Key Words: telepathology, pathology, Internet, telehealth, telemedicine

\section{Introduction}

Telepathology is "the electronic transmission of pathological images... from one place to another for interpretation and diagnosis."1 It is one of the more developed fields of telemedicine. Its successful practice commonly requires human, organizational, and technology components to be in place and synchronized. Technology components usually comprise basic processing of specimens

Presented at the HealthNet 2020, the 20th year anniversary celebration of Internet in the Philippines, March 27, 2014, Ateneo Java Wireless Center, Diliman, Quezon City.

Corresponding author: Elizabeth Arcellana-Nuqui, MD

The Medical City

Ortigas Avenue, Pasig City

Telephone: +6329887000 or 9881000 local 6126

Email: betheyan@gmail.com into pathology slides at remote sites, the ability to digitize and transmit these through the Internet to a pathologist expert, and to establish secure communications between corresponding physicians. The organizational component consists of agreements between health facilities to collaborate for better quality of care while human component is made up of the appropriate knowledge, skills, and attitudes to employ technology for remote telepathology consultation. Equipment needed are microscope cameras (or slide scanners), computers, and adequate Internet connectivity.

There are two basic methods for telepathology. Storeand-forward method entails capturing and uploading representative images from the microscope slides while the real-time method requires transmission of live video clips of the slides. For both cases, a secure communication system is created between corresponding physicians.

More recent equipment now allow senders and experts to control the microscope, select images and view incoming images in real-time using robots. Cost is a major drawback in the dissemination of this modality. Another more affordable option is whole slide imaging (WHI) or virtual microscopy which makes it possible to digitize an entire slide and produce a virtual slide for viewing using computer monitors. While these applications and tools exist, telepathology it is still not a global practice most likely because of cost. Current implementations are highly subsidized by academic institutions or governments or occur in more developed countries. The WHO Global Observatory for eHealth (2008) reported "an overall lack of awareness as to what eHealth tools and services already exist worldwide."2 More recent work by Park $^{3}$ confirms that telepathology is challenging and fraught with failures even in well-resourced developed countries. He attributes these to poor infrastructure and strict regulatory policies. Wamala ${ }^{4}$ confirms the same issues in a meta-analysis of telemedicine in Africa.

The first routine telepathology system was implemented at the University of Tromsø, Norway, in $1989^{5}$ with the required hardware, dedicated software, and special microwave transmission links, and innovator-pathologists. But subsequent adoption was slow because of the high cost of equipment and the inability of many people to replicate the system. In 1990, "the personal computer provided more 
adequate performance at low cost for image display quality and speed, and the development of video technology resulted in high quality images that were more affordable;"4 microscopes also became more affordable. By mid-1990s, all hardware necessary to implement a telepathology system became available at a more reasonable cost. ${ }^{6}$ In 1995, the Armed Forces Institute of Pathology (AFIP) developed a store-and-forward system based on non-proprietary, off-theshelf hardware, and open Internet protocols (file transfer protocol, e-mail attachments) that served many locations worldwide, including the Philippines. ${ }^{7,8}$

\section{Telemedicine: Opportunities in the Philippines}

In the Philippines, an archipelagic country of 7,107 islands in Southeast Asia, the first reported use of modern telepathology consult was in 1997. Pathologists are specialists in Laboratory Medicine who evaluate tissue biopsy, cytology and other patient samples (body fluids and cell aspirates) removed during surgery or image-guided procedures, to diagnose diseases in patients. This function in many cases initiates the treatment and management of the patient's condition. The input of the primary care physician in providing the patient's history is essential in the practice of pathology. Pathology expertise requires many years of training in the morphologic, biochemical and more recently, the molecular alterations in diseases.

There is a maldistribution of pathologists in the Philippines; just like many highly-specialized areas of medicine, pathologists are concentrated in urban areas. Currently, the pathologist-to-patient ratio is estimated at 1:167,000. Of the more than 600 pathologists on the 2010 roster of the Philippine Society of Pathologists, 55\% are based within the National Capital Region. Only three pathologists are registered in the Mindoro, Marinduque, Romblon, Palawan (MIMAROPA) region, four in Zamboanga and none in the Autonomous Region of Muslim Mindanao (ARMM). The Philippine Society of Pathologists directory reveals that the majority practice in Metro Cebu or Metro Bacolod in the central Visayas regions. The status of at least 83 pathologists is unknown (Table 1 ).

Where confronted with a challenging case, pathologists consult other pathologists. In the 1990's, this was usually done by sending the glass slides of tissue samples through the regular postal system then waiting for pathology reports again through the same route. After samples have been thoroughly studied, the pathologist renders a diagnosis and transmits the pathology report to the referring pathologist or attending physician. This entire process took between one to four weeks. In the late 1990s, there was only one courier service in the country, a service which could cut in half the turn-around-time. However, this meant it could still take about two weeks for a definitive diagnosis. This slow process delayed clear-cut diagnosis particularly for patients in whom cancer was suspected and where definitive treatment could not proceed without histologic confirmation. This was the setting when the first telepathology consult took place in the Philippines in 1997.

Table 1. Geographic distribution of pathologists per region (Philippine Society of Pathologists, 2010)

\begin{tabular}{cc}
\hline Region & Number of Registered Pathologists \\
\hline 1 & 10 \\
2 & 11 \\
3 & 27 \\
5 & 6 \\
6 & 31 \\
7 & 33 \\
8 & 9 \\
9 & 6 \\
10 & 7 \\
11 & 10 \\
12 & 4 \\
13 & 7 \\
4 A & 23 \\
$4 B$ & 3 \\
ARMM & 0 \\
CAR & 18 \\
NCR & 252 \\
Unknown Status & 83 \\
\hline
\end{tabular}

\section{The First Internet-based Telepathology Consult}

In 1997, links with medical and health informatics experts at the Armed Forces Institute of Pathologists (AFIP) and the establishment of Internet access at the University of the Philippines Manila (UPM) created the environment for remote pathology consultation that had become the first telepathology consult in the Philippines. The AFIP was a major training and referral center for anatomic and surgical pathology, and published three series of fascicles on tumors of all organ sites, written by the most illustrious academic pathologists of their time. Before the AFIP was closed in 2011 along with other military medical institutions, ${ }^{9}$ these fascicles served as the reference books for pathology residents- and fellows- in training throughout the world. The objective of this Philippine telepathology consult was to demonstrate that a consultant pathologist could render an opinion based on the evaluation of microscopic images transmitted through the Internet from the Philippines. Telepathology necessitates the capture of representative images of the tissue specimen that are of optimal resolution and magnification adequate to make a thorough evaluation of the specimen and provide an expert opinion.

\section{Consultation process}

The first telepathology consult between the UPM and the AFIP occurred on 16 January 1997 (Figure 1). In order to protect the privacy of the patient, de-identified representative images from a tissue slide were sent as email attachments via Internet to the AFIP. Although the images of the slide could no longer be retrieved from the AFIP, email logs show the diagnosis from the referring pathologist as "aggressive angiomyxoma," a vascular soft tissue tumor. The 
AFIP rendered a diagnosis: "Vascular Mesenchymal Neoplasm," within 48 hours of the email consult with a comment to "Please send glass slide." The generic diagnosis and the comment highlighted the limitations of electronic consultations in the 1990's with regard to the quality of image resolution and capture.
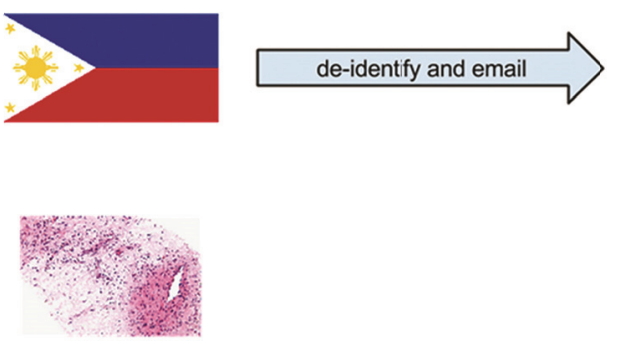

Capture images
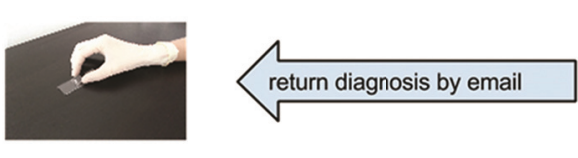

Prepare slides

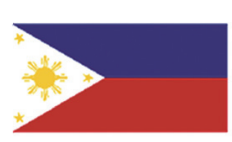

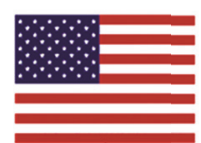

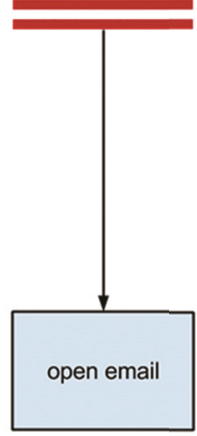

Figure 1. Process Map of the first Telepathology consult between the UPM and the AFIP, January 1997.

This was considered a successful telepathology consult. It was able to demonstrate that referring pathologists (source) can capture representative images electronically and transmit them anonymously to remote experts. These experts in turn can make a diagnosis based on adequately selected and clear images using electronic mail.

\section{Lessons from the First Consult}

In a country challenged with geographic disparities and maldistribution of experts, it seems obvious that health solutions like telepathology should become mainstream.

However, nearly two decades later, the service has not scaled to serve remote underserved areas as expected. These issues can be divided into three areas as described by Yusof ${ }^{10}$ - human, organizational and technology-fit (HOTfit) factors.

\section{Human Factors}

Expert Consultants Must Be Ready and Willing

The telepathology case was a situation of second opinion consultation between pathology colleagues; this was the concept of the implementers back then. A diagnosis had already been rendered at UP-PGH. The expert/specialist referring (source) pathologist was able to capture areas showing characteristic morphologic features of the specimen which were then relayed to the remote consultant. This selection process contributed to the successful diagnosis of the remote pathologist in AFIP. On top of this mastery of pathology, the participating pathologists were also willing to learn the mechanics of the technology - using the Internet, compressing images, et cetera - in order to provide appropriate consult services successfully. In essence, there should be a willingness of both parties to collaborate and resolve the diagnosis for the difficult case. While adequate technical support was available for the first telepathology consult, the same support was not institutionalized and made available for subsequent ones. This meant that pathologists who wanted to do telepathology thereafter had to perform all tasks for themselves.

\section{Conventional methods for training pathologists}

The UP College of Medicine-Philippine General Hospital offers a pathology residency program which admits physician trainees from all over the country, and which also facilitates the graduates' return to their respective areas. At present, the UP-PGH Pathology graduates are serving in Cagayan Valley and Kalinga-Apayao in the far north, Bicol, Capiz, Bohol, Leyte, Misamis, Cotabato, Bukidnon in the Visayas and Mindanao - practically all regions of the Philippines, with few exceptions. Currently, telepathology is not embedded in the training.

In 1997 as in the present, pathology training requires viewing of the specimen slide done simultaneously, side-byside and in real-time, by the pathologist (trainer) and the pathology resident physician (trainee). Imagining telepathology for the Philippines at that time, this was also the preferred methodology: real-time and simultaneous viewing of the glass slide by the referring pathologist (based in the provinces) and the consultant pathologist (based in the metro areas).

The number of optical microscope heads available in the sign-out rooms in PGH and the UP College of Medicine Department of Pathology was a rate-limiting step in pathology training. Pathologists "sign out" a specimen when the final official diagnosis is rendered documented in a report, which is physically and officially signed by the pathologist, not the trainee.

\section{Organizational Factors}

De-monopolizing Medical Knowledge

For many years, pathology specialty and subspecialty expertise have been concentrated in academic institutions and tertiary care centers. This is the opportune time for pathologists to come to extend their faculties to provide service to the people, especially in remote areas, a response to Arora and colleagues ${ }^{11}$ appeal for "de-monopolizing medical knowledge."

Telepathology is for remote primary diagnosis, remote referral to specialist-pathologists, remote teaching, remote presentation of post-mortem or microscopic findings, quality assurance image circulation and feedback, and consensus diagnosis for pathological review in clinical trials. ${ }^{9}$ Telepathology can help general pathologists who 
come across unconventional cases in smaller labs doing routine histopathology in remote regions without specialistpathologist (pathologists who have undergone subspecialty training in specific organ systems). Without handing over specimen blocks to the expert, a real-time consultation and diagnosis can take place between two pathologists who are hundreds of kilometers apart, using telepathology. This can enhance the teaching-learning experience better than conventional consultation as the local pathologist observes and gets the opportunity to understand the diagnostic approach taken by the expert. Viewing images remotely reduces the expense and travel time of the referring pathologist who requires a second opinion or concurrence.

As the Internet bandwidth expands and becomes more affordable, and with new software being developed and made available, current medical practice is unable to keep pace with the explosion in medical knowledge. Specialized medical knowledge is confined largely to academic medical centers, teaching hospitals, and to specialists in major cities.

At present, primary care clinicians on the front lines of patient care are disconnected from academic centers. To bridge this gap, medical knowledge must be demonopolized. Platforms for collaborative practice amongst clinicians need to be created.

\section{Governance}

Recent research on telehealth and telemedicine ${ }^{11}$ reveals that these services, by virtue of the many components involved in its successful operation, often succumb to complexity. As such, governance plays a factor in the success of an extended telemedicine service such as telepathology. In the first telepathology consult, the governance had been simple due to its nature of research. Only the source pathologist and the remote expert were involved and their institutional arrangements were not necessary. In a production mode telepathology service, governance will need to be clearly established to manage the complexities of implementation.

Establishing a formal telepathology service and having it recognized Initiated in 1993, AFIP 8 already had extensive experience with telepathology and had established international leadership in its practice. When the proposal to make a telepathology referral to AFIP was made to the Filipino pathologist, the idea was immediately accepted and there was no doubt that the whole referral process would be of high quality. This fact had been implicit but important to the success of the first consult.

\section{Process}

Surgical pathology - where tissue samples from tumors or suspected cancerous lesions - could not be done in most primary care settings. For surgical pathology, surgeons are trained to excise masses appropriately, including margins and lymph nodes. Tissue preparation needs special handling - fixation and processing through a series of alcohols, embedding in paraffin blocks, cutting thin sections using microtomes, staining, et cetera - by trained medical technologists in an adequately-equipped laboratory. Such equipment is not available in primary care settings. What was demonstrated in 1997 is an example of a surgical pathology consult, where the working diagnosis was an aggressive soft tissue tumor of blood vessels from slides that were processed in a tertiary care hospital laboratory.

\section{Technology Factors \\ Hardware}

The altruism, interest, and commitment of the participating pathologists, institutions, and agencies allowed the initial demonstration of telepathology at that time. But these were insufficient to propel telepathology as a practice and service in the Philippines. Limitations are myriad.

At that time, the expense of hardware was prohibitive to set up at the UPM Pathology Laboratory. To take an image from a slide required a special type of monocular microscopy camera unit, which UPM did not have at that time.

\section{Connectivity}

In 1997, the Internet link at the University of the Philippines was still very slow. Although the server was called "kulog," which literally meant "thunder," it was ironically extremely limited. Bandwidth to the entire country was a mere $64 \mathrm{kbps}$ shared among five of the largest universities of the Philippines (the University of the Philippines, Ateneo de Manila, De La Salle, University of Santo Tomas, all in Manila, as well as St. Louis University in Baguio City, located 250 kilometers north of Manila in the Mountain Province.) This was really the early age of Internet in the Philippines. The only practical method of sending high-resolution images with that bandwidth was electronic mail.

\section{Moving Forward}

The Hub and Spokes of Telepathology

It is important to realize that pathology is at the hub of an effective telehealth system. From a diagnostic work-up done in a clinical laboratory (hematology, clinical chemistry, immunology, microbiology, molecular pathology) to the tissue confirmation of a surgical biopsy, from an accurate and timely pathologic diagnosis, emanate all the options for management and care of patients. Like the AFIP, a center (hub) for telepathology is important in creating credibility for the service.

A referral network (spokes) of telepathologists can encourage pathology practice outside the National Capital Region and other metropolitan areas, specialist pathologists supporting general pathologists. Telepathology 
consultations and referrals will result in increased confidence and enhancement of pathologists' diagnostic skills and proficiency, which in turn will help solve the geographic maldistribution of these crucial services. This would uplift and upgrade the quality of care in remote places, reduce turn-around-times for cytology and pathology reports which will lead to early diagnosis and treatment of cancer and other serious illnesses, and thereby improve the quality of life and survival of our patients.

Pathologists in the Philippines today can consider remote pathology training using IP (Internet Protocol) videoconferencing tools or web-casting for remote pathology education during specimen sign-outs. Practitioners should explore the use of IP videoconferencing for frozen sections and real-time primary diagnosis of surgical cases as a teleconsultation application in medicine and healthcare. Remote pathologists could also show a challenging case remotely, where interactions are captured and archived on web servers so others can learn from interesting cases. In such instances, continuing medical education credits can be awarded.

Telepathology can have an important role in education and training: online cases and images are excellent tools of education, supplementing standard books. Video clips from case conferences can be part of routine training; virtual slide technology can reduce the number of microscopes needed in class. Telepathology is an excellent tool to distribute material from small biopsies, especially those cases that appear to defy typical diagnosis, to many other pathologists. Participants can see and discuss the same images and maintain a record for future reference. Telepathology can also be part of an external quality assurance program to measure and improve the proficiency of pathologists. Other telepathology tools are iPath, $^{12}$ Whole Slide Imaging Repository, ${ }^{13}$ Real-time Telepathology Imaging System, ${ }^{14}$ Physically Virtual Slide ${ }^{13}$ used in virtual telepathology education congresses or Virtual Congresses, and others.

Ultimately, telepathology is the practice of pathology over a distance. Therefore, to ensure a successful program, the subject matter experts must establish how such a practice should be optimized for use in the country. The human, organizational, and technology factors must be addressed to enable the service to scale nationally.

The topic for our paper on telepathology was occasioned by the anniversary lecture on the first transPacific telepathology consultation from the Department of Pathology of the University of the Philippines College of Medicine to the Armed Forces Institute of Pathology of the United States in 1997. The lecture was delivered during a symposium organized by Dr. Alvin B. Marcelo, President of the Medical Informatics Society, at the Ateneo de Manila University in Loyola Heights, Quezon City.

This look-back to twenty years ago reveals that the practice of pathology in the Philippines is still constrained by geography and topography, maldistribution of pathologists who are mostly clustered around the urban areas and metro-cities, and the lack of a functional network for consultation on difficult diagnostic problems. Even in the 21st century when telepathology equipment and software, and efficient electronic systems are more affordable, the impediments seem to be the resistance among pathologists over concerns dealing with trust, fees, and accountability. The implementation of a professional telepathology network would raise the standard of pathology practice, enhance diagnostic accuracy and turn-around time for earlier diagnosis and treatment of cancer to improve survival and Quality of Life for our Filipino patients.

\section{References}

1. Express Healthcare. Telepathology: The Future of Telepathology [Online]. 2008 [cited 2014 April]. Available from http://healthcare. financialexpress.com/200806/labwatch03.shtml.

2. World Health Organization-Global Repository on eHealth. eHealth Tools \& Services: Needs of the Member States. Report of the WHO Global Observatory on eHealth [Online]. 2006 [cited 2014 April]. Available from http://www.who.int/goe/publications/ehealth_tools_services/en/.

3. Park S, Parwani AV, Aller RD, et al. The history of pathology informatics: A global perspective. J Pathol Inform. 2013; 4:7.

4. Wamala DS, Augustine K. A meta-analysis of telemedicine success in Africa. J Pathol Inform. 2013; 4:6.

5. Weinstein RS. Telepathology comes of age in Norway. Hum Pathol. 1991; 22(6):511-3.

6. Wells CA, Sowter C. Telepathology: a diagnostic tool for the millennium? J Pathol. 2000; 191(1):1-7.

7. Fontelo PA. Telepathology at the AFIP. Telemed Today. 1996; 6:17-22 a.

8. Mullick FG, Fontelo P, Pemble C. Telemedicine and telepathology at the Armed Forces Institute of Pathology: history and current mission. Telemed J. 1996; 2(3):187-93.

9. Genome web. The Sample. Molecular Tools in the Clinical Lab. ASCP Mourns Closure of the Armed Forces Institute of Pathology [Online] 2011 [cited 2014 April]. Available from http://www.genomeweb. com/blog/ascp-mourns-closure-armed-forces-institute-pathology.

10. Yusof MM, Kuljis J, Papazafeiropoulou A, Stergioulas LK. An evaluation framework for Health Information Systems: human, organization and technology-fit factors (HOT-fit). Int J Med Inform. 2008; 77(6):386-98.

11. Jarvis L, Stanberry B. Teleradiology: threat or opportunity? Clin Radiol. 2005; 60(8):840-5.

12. iPath Community. iPath [Online]. 2011 [cited 2014 April]. Available from http://www.ipath-network.com/moldova/.

13. Digital Pathology Association. Whole Slide Imaging Repository [Online]. 2014 [cited 2014 April]. Available from https://digitalpathology association.org/whole-slide-imaging-repository.

14. Meyer Instruments. Real-time Telepathology Imaging System [Online].2012 [cited 2014 April]. Available from http://realtime telepathology.com/rtis-accessories. 